Bazyli DEGÓRSKI O.S.P.P.E.*

\title{
LA PREFAZIONE DI SAN GIROLAMO ALLA CONTINUAZIONE DELLA CRONACA DI EUSEBIO DI CESAREA
}

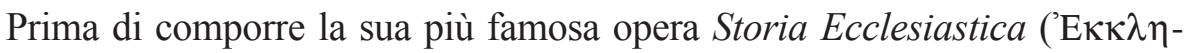

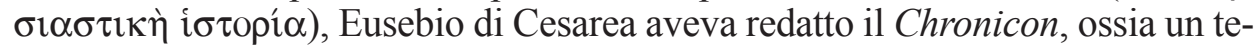
sto storiografico che riassume la cronologia universale e biblica. La versione originale greca dell'opera è andata perduta, ma ne restano una traduzione completa in lingua armena ${ }^{1}$ e una parziale in latino, realizzata proprio da san Girolamo.

Dopo aver presentato una sintesi di storia orientale e greca in base alle fonti scritturistiche, nella seconda parte dell'opera Eusebio fornisce una tavola sincronica a più colonne, che copre il periodo temporale da Abramo (2016 a.C.) sino all'anno 303 d.C.

Proprio questa seconda parte del Chronicon eusebiano (noto anche con il titolo Temporum liber o breviarium) fu tradotta da san Girolamo e da lui stesso continuata sino all'anno 378 dopo Cristo. Il Dalmata lesse il Chronicon di Eusebio e lo tradusse nel 380 circa, durante il suo soggiorno a Costantinopoli ${ }^{2}$.

Nonostante numerosi errori e incongruenze, ripresi dal testo greco di Eusebio, ma anche commessi dallo stesso Girolamo, quest'opera storica è molto utile ancora oggi e, nel passato, ispirò il lavoro storiografico degli autori

* Prof. dr hab. Bazyli Degórski, O.S.P.P.E. - professore di patrologia e di teologia dogmatica dell'età patristica presso la Pontificia Università S. Tommaso d'Aquino a Roma, e professore di patrologia e di antropologia patristica alla Pontificia Facoltà Teologica "Teresianum" a Roma; procuratore generale presso la Santa Sede; email: osppe.roma@gmail.com.

${ }^{1}$ Cf. Eusebii Pamphili Caesariensis Episcopi, Chronicon bipartitum, nunc primum ex Armeniaco textu in Latinum conversum, adnotationibus auctum, Graecis fragmentis exornatum, ed. J.B. Aucher, I-II, Venetiis 1818 (versione armena e traduzione latina dall'armeno).

${ }^{2}$ Cf. J. Gribomont, Le traduzioni. Girolamo e Rufino, in: Patrologia, vol. 3: Dal Concilio di Nicea (325) al Concilio di Calcedonia (451). I Padri latini, ed. A. Di Berardino, Casale Monferrato 1978, 217. Secondo A. Grisart (La Chronique de Saint Jérôme. Le lieu et la date de sa composition, "Hélikon" 2 (1962) 248-258), san Girolamo la scrisse a Treviri, negli anni 368-371! 
paleocristiani che la continuarono, quali ad esempio: Prospero di Aquitania ${ }^{3}$, Cassiodoro $^{4}$, o Vittore di Tunnuna ${ }^{5}$.

\section{BREVE ANALISI DELLA PREFAZIONE DI SAN GIROLAMO ALLA TRADUZIONE DELLE CRONACHE DI EUSEBIO DI CESAREA}

San Girolamo inizia l'introduzione alla sua traduzione delle Cronache di Eusebio di Cesarea salutando i suoi compagni, conosciuti al tempo del suo soggiorno nel monastero di Aquileia: Vincenzo e Gallieno, ai quali dedica la propria opera ${ }^{6}$.

Nel primo capitolo della Praefatio, il Dalmata si appella a dei suoi "predecessori" che traducevano dal greco in latino opere importanti, scritte sia in prosa che in versi. San Girolamo, però, mette in rilievo numerose difficoltà che si fanno sentire in queste ardue imprese che tutti i traduttori del mondo devono affrontare. Spessissimo, il traduttore è costretto a "storpiare" l'originale per renderne il vero senso, conformemente alla lingua alla quale il testo viene tradotto?:

"È stata da lungo tempo abitudine degli uomini dotti esercitare le loro menti attraverso la resa in latino dei libri dei greci e, cosa ancor più difficile, tradurre i poemi di illustri personaggi aggiungendo la necessità di farlo in versi. È anche per questo motivo che il nostro Tullio ${ }^{8}$ tradusse alla lettera

${ }^{3}$ Prosper Aquitanus, Epitoma Chronicorum, PL 51, 535-606; ed. T. Mommsen, MGH Auct. Ant. IX, Berolini 1892, 385-485; Additamenta, tamże, s. 486-499, PLS 3, 147-148. Cf. A. Hamman, Scrittori della Gallia, in: Patrologia, vol. 3, s. 525-526.

${ }^{4}$ Cassiodorus, Chronica, PL 69, 1213-1247; ed. T. Mommsen, MGH Auct. Ant. XI, Berolini 1894, 109-161. Cf. U. Pizzani, Scrittori dell'Italia, in: Patrologia, vol. 4: Dal Concilio di Calcedonia (451) a Beda. I Padri latini, ed. A. Di Berardino, Genova 1966, 220.

${ }^{5}$ Victor Tunnunensis, Chronica, PL 68, 941-962; ed. T. Mommsen, MGH Auct. Ant. XI 163206; ed. C. Hartmann, CCL 173A, Turnhout 2001. Cf. anche M. Simonetti, Letteratura cristiana d'Africa, in: Patrologia, vol. 4, s. 38-40; F. Scorza Barcellona, Vittore di Tunnuna, NDPAC III 56785679. Fra i "continuatori" dell'opera geronimiana potrebbe essere annoverato forse anche Agapito di Gerapoli. Cf. Agapito di Gerapoli. Storia universale, ed. B. Pirone, Milano 2013.

${ }^{6}$ Cf. Hieronymus, Praefatio in Eusebii Caesariensis Chronicon 1, PL 27, 33A: "Eusebius Hieronymus Vincentio et Gallieno suis salutem".

${ }^{7}$ La traduzione italiana di questo testo di san Girolamo è di Bazyli Degórski.

${ }^{8}$ Marco Tullio Cicerone. Nacque il 3 gennaio del 106 a.C. in località Ponte Olmo, oggi nel territorio di Sora, ma all'epoca nel municipio di Arpinum, antica città di collina fondata dai Volsci, a $100 \mathrm{~km}$ a sud di Roma, e morì assassinato, a Formia, il 7 dicembre 43 a.C., da alcuni sicari (inviati da Marco Antonio), aiutati da un liberto di nome Filologo. Grazie a Cicerone, esimio amante anche della cultura greca, i Romani acquisirono una migliore conoscenza della filosofia. Egli, infatti, si impegnò ad escogitare il corrispondente vocabolo, in latino, per tutte le parole peculiari del linguaggio filosofico greco. 
interi libri di Platone ${ }^{9}$ e, dopo aver pubblicato Arato ${ }^{10}$ (che già [può considerarsi] romano) in versi esametri, si divertì con l'Economico di Senofonte ${ }^{11}$. In questa opera, quel dorato fiume dell'eloquenza si scontra più e più volte con ostacoli contro i quali le sue acque si infrangono e schiumano a tal punto che, a meno di non avere familiarità con l'originale, non si crederebbe di ascoltare parole di Cicerone. È, infatti, difficile seguire il filo di un'altra persona e mantenersi entro i limiti. È difficoltoso, in una traduzione, mantenere l'espressività e la grazia. Una parola ha forzosamente espresso un determinato pensiero; io non ho parole per esprimere il significato e, mentre mi sforzo di rendere il senso, posso divagare a lungo percorrendo, in realtà, solo una piccola porzione del mio viaggio. Occorre prendere consapevolezza delle giravolte della trasposizione, le variazioni dei casi, la diversità delle figure e, infine, la peculiarità, ovvero l'origine idiomatica, per così dire, della lingua. Se traduco letteralmente, suona assurdo. Se sono costretto a cambiare o l'ordine delle parole o le parole stesse, sembro aver rinunciato al dovere di un traduttore" 12 .

${ }^{9}$ Platone (427-348 a.C.), discepolo di Socrate. Marco Tullio Cicerone tradusse due dialogi di Platone: il Timeo e il Protagora.

${ }^{10}$ Arato ("A $\alpha \tau$ (o), nato a Soli in Cilicia, nel 310 a.C. circa - morto nel 240 a.C. circa, fu un poeta greco del primo Ellenismo. Di Arato ci resta solamente il suo capolavoro, il poema didascalico Fenomeni, di 1154 esametri, di contenuto astronomico. L'opera volge in versi la materia già presentata dal matematico e astronomo Eudosso di Cnido, discepolo di Archita di Taranto e di Platone. La traduzione latina di Cicerone, sempre in esametri, gli Aratea, è forse l'opera di maggior successo del "Cicerone poeta".

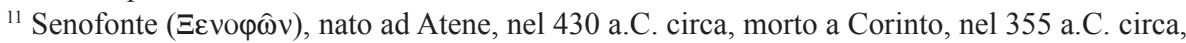
fu uno storico e mercenario ateniese. Di lui ci sono pervenute tutte le opere complete. Oltre che da Platone, su Senofonte ci pervengono da molte notizie e particolari riguardanti la vita e i detti di Socrate. Senofonte scrisse le seguenti opere storiche: Anabasi, Ciropedia, Elleniche, Agesilao, Consti-

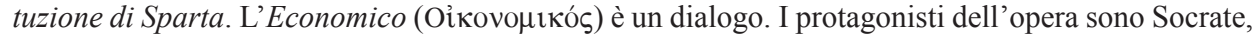
maestro di Senofonte e il giovane Critobulo. La prima parte del dialogo rispecchia il titolo (leggi per il governo della casa) ma, in seguito, si passa al racconto da parte del filosofo di una conversazione avuta con un ricco proprietario terriero, Isimaco, sul modo di amministrare i beni. L'opera è anche una lode della vita contadina. Cicerone la tradusse in latino.

${ }^{12}$ Hieronymus, Praefatio in Eusebii Caesariensis Chronicon 1, PL 27, 33-35: "Vetus iste disertorum mos fuit, ut exercendi ingenii causa Graecos libros Latino sermone absolverent et, quod plus in se difficultatis habet, poemata inlustrium virorum addita metri necessitate transferrent. Unde et noster Tullius Platonis integros libros ad verbum interpretatus est et cum Aratum iam Romanum hexametris versibus edidisset, in Xenophontis Oeconomico lusit. In quo opere ita saepe aureum illud flumen eloquentiae quibusdam scabris et turbulentis obicibus retardatur, ut, qui interpretata nesciunt, a Cicerone dicta non credant. Difficile est enim alienas lineas insequentem non alicubi excidere, arduum, ut quae in alia lingua bene dicta sunt eundem decorem in translatione conservent. Significatum est aliquid unius verbi proprietate: non habeo meum, quo id efferam, et dum quaero implere sententiam, longo ambitu vix brevis viae spatia consumo. Accedunt hyperbatorum amfractus, dissimilitudines casuum, varietas figurarum, ipsum postremo suum et, ut ita dicam, vernaculum linguae genus. Si ad verbum interpretor, absurde resonat: si ob necessitatem aliquid in ordine, in sermone mutavero, ab interpretis videbor officio recessisse". 
Nel secondo capitolo della Praefatio, san Girolamo si rivolge, quindi, a Vincenzo e Gallieno e, ovviamente, a tutti i futuri lettori della sua traduzione, chiedendo a loro di servirsi di essa "con l'occhio dell'amico più che con quello del critico", a maggior ragione che, come spiega il Dalmata, egli tradusse l'opera Eusebiana, dettandola ai suoi amanuensi, "con grande rapidità". Inoltre, lo stesso testo di Eusebio fu difficile da intendere, quindi anche da tradurre. San Girolamo menziona qui pure diverse traduzioni dell'Antico Testamento, fatte dall' ebraico al greco dai Settanta Interpreti, da Aquila, Simmaco e da Teodozione, i quali, svolgendo quest'ardua impresa, raggiunsero diversi risultati delle loro fatiche, imprimendo con le loro traduzioni "un carattere totalmente differente alla medesima opera":

"Perciò, mio caro Vincenzo, e tu, Gallieno, parte della mia anima ${ }^{13}$, vi supplico, qualunque sia il valore di questo affrettato lavoro, di leggerlo con l'occhio dell'amico più che con quello del critico. E chiedo questo ancor più ardentemente perché, come sapete, ho dettato ai miei amanuensi con grande rapidità; e quanto tale opera sia difficile lo testimoniano i documenti dei Libri Sacri che, pubblicati dai Settanta Interpreti ${ }^{14}$, non conservano lo stesso sapore nella lingua greca. È stato questo che ha spinto Aquila ${ }^{15}$ e Simmaco ${ }^{16}$

${ }^{13}$ Cf. Quintus Horatius Flaccus, Carmina I 3, 5-8.

${ }^{14}$ La versione dei Settanta (Septuaginta in latino) è la traduzione della Sacra Scrittura in lingua greca. La cosiddetta Lettera di Aristea (o Lettera dello pseudo Aristea) a Filocrate (appartenente alla pseudoepigrafia ellenistica del II secolo a.C.) è verosimilmente il primo testo che riguardi le origini di questa versione greca della Bibbia. Secondo questa Lettera, il re egiziano Tolomeo II Filadelfo (285-246 a.C.) commissionò alle autorità religiose ebraiche di Gerusalemme, per la biblioteca di Alessandria, una traduzione in greco del Pentateuco. Il sommo sacerdote Eleazaro, quindi, nominò 72 eruditi ebrei (secondo altre versioni 70) che andarono ad Alessandria. In seguito, stando nell'isola di Faro, essi completarono in modo indipendente la traduzione (e ciò in 72 giorni) e, comparando tra di loro le versioni, costatarono con meraviglia che le loro traduzioni erano identiche. Alcuni però riferiscono che fossero soltanto cinque i traduttori, settanta invece sarebbero stati i membri del sinedrio che approvarono la corrispondenza fra la traduzione greca e l'originale ebraico. Per le Chiese orientali di lingua greca, la versione dei Settanta costituisce tuttora la traduzione liturgica ufficiale dell'Antico Testamento.

${ }^{15}$ Aquila di Sinope, verso il 130 tradusse in greco, in modo del tutto letterale, l'originale ebraico della Sacra Scrittura. Era un proselita della religione mosaica e discepolo di Rabbì Akiba (morto nel 135 circa). Si dice che la traduzione di Aquila fosse usata nelle sinagoghe al posto della Septuaginta. Origene la incorporò nei suoi Hexaplà.

${ }^{16}$ Simmaco l'Ebionita, vissuto nel tardo II secolo d.C., fu l'autore di una delle versioni greche dell'Antico Testamento che venne inclusa da Origene nei suoi Hexaplà e Tetraplà. I frammenti della traduzione di Simmaco, presenti in ciò che rimane degli Hexaplà, testimoniano l'eleganza dello stile greco di Simmaco, che fu apprezzata da san Girolamo. Simmaco scrisse anche dei Commentari, gli Hypomnemata (cf. Eusebius Caesariensis, HE VI 17; Hieronymus, De viris inlustribus 54), per noi ora perduti. Li compose, pare, per confutare la versione canonica greca del Vangelo di san Matteo. Con tutta probabilità, si tratta della stessa opera menzionata da Ebed Jesu, con il titolo De distinctione praeceptorum. Origene afferma di aver ottenuto queste ed altre copie dei Commentari biblici di Simmaco da una certa Giuliana, che le aveva ereditate da Simmaco stesso (cf. Eusebius Caesarien- 
e Teodozione ${ }^{17}$; e il risultato dei loro sforzi fu quello di imprimere un carattere totalmente differente alla medesima opera; uno tentò di tradurre parola per parola, un altro il significato generale, mentre il terzo si adoperò per evitare di divergere eccessivamente dagli antichi. Una quinta, una sesta e una settima edizione $^{18}$, sebbene non si sappia a quali autori debbano attribuirsi, esibiscono una così amena varietà che, a dispetto dell'anonimato, hanno conseguito una certa autorevolezza. Perciò, alcuni si spingono a considerare le Sacre Scritture come dure in qualche modo e stridenti all'orecchio; ciò si origina dal fatto che le persone cui mi sto riferendo non sono al corrente che le scritture in questione sono una traduzione dall'ebraico, per cui, restando in superficie e non alla sostanza, essi sobbalzano di fronte al vestito sciatto prima di scoprire il corpo bello che il linguaggio veste" ${ }^{\text {. }}$.

sis, HE VI 17). Sant'Epifanio di Salamina scrive che Simmaco era un Samaritano, il quale aveva litigato con i suoi correligionari e si era convertito all'Ebraismo (cf. De mensuris et ponderibus 16),

${ }^{17}$ Teodozione, un erudito ebreo di cultura greca del I o del II secolo, tradusse dall'originale ebraico in greco l'Antico Testamento e questa sua versione fu inserita, verso il 240, da Origene dell'ultima colonna dei suoi Hexaplà. Sant'Ireneo di Lione è il primo a menzionarlo e lo chiama "Teodozione di Efeso" (Adversus haereses 3, 21). Tale prima testimonianza di sant'Ireneo viene ripetuta pure da Eusebo di Cesarea (HE V 8).

${ }^{18}$ Con tanta probabilità si tratta qui almeno delle traduzioni fatte da Esichio di Gerusalemme e da san Luciano di Antiochia, anche se pare curioso che san Girolamo non parli espressamente di loro. Esichio di Gerusalemme (non conosciamo la data della sua nascita; morì probabilmente verso il 433), fu un presbitero cristiano ed un esegeta. Nulla di sicuro conosciamo della sua vita. Parte dei suoi scritti sono andati perduti, mentre in parte ci sono stati tramandati come opere di altri autori. J.-P. Migne ha pubblicato sotto il titolo "Esichio, presbitero di Gerusalemme" le opere di vari autori di nome "Esichio" (PG 93, 787-1560), senza alcun riguardo per l'ordine. Quindi, circa la metà di questo materiale va scartata. San Luciano di Antiochia (nato a Samosata, nel 235 circa, e morto a Nicomedia, il 7 gennaio 312) è stato teologo e martire. Considerando prevalentemente la sola interpretazione letterale della Bibbia, egli sottolineò il bisogno di esattezza testuale e, quindi, intraprese la revisione del Septuaginta nell'originale ebraico. Questa sua revisione della Sacra Scrittura fu ampiamente utilizzata nel IV secolo (cf. Hieronymus, De viris inlustribus 77; idem, Praefatio in librum Paralipomenon de graeco emendato; idem, Apologia contra Rufinum 36; idem, Epistula 106). San Luciano fece pure una revisione del Nuovo Testamento.

${ }^{19}$ Hieronymus, Praefatio in Eusebii Caesariensis Chronicon 2, PL 27, 35-36: "Itaque, mi Vincenti carissime, et tu, Galliene, pars animae meae, obsecro, ut, quidquid hoc tumultuarii operis est, amicorum, non iudicum animo relegatis, praesertim cum et notario, ut scitis, volocissime dictaverim et difficultatem rei etiam divinorum voluminum instrumenta testentur, quae a septuaginta interpretibus edita non eundem saporem in Graeco sermone custodiunt. Quam ob rem Aquila et Symmachus et Theodotio incitati diversum paene opus in eodem opere prodiderunt, alio nitente verbum de verbo exprimere, alio sensum potius sequi, tertio non multum a veteribus discrepare. Quinta autem et sexta et septima editio, licet quibus censeantur auctoribus ignoretur, tamen ita probabilem sui diversitatem tenent, ut auctoritatem sine nominibus meruerint. Inde adeo venit, ut sacrae litterae minus comptae et sonantes videantur, quod diserti homines interpretatas eas de Hebraeo nescientes, dum superficiem, non medullam inspiciunt, ante quasi vestem orationis sordidam perhorrescant quam pulchrum intrinsecus rerum corpus inveniant". 
Il Dalmata continua fornendo degli altri esempi per mettere in rilievo delle difficoltà, specialmente quelle che incontrano traduttori di testi di stampo poetico:

"Infatti, che cosa mai può essere più musicale del Salterio, come [gli scritti] del nostro Flacco ${ }^{20}$ e del greco Pindaro ${ }^{21}$, ora si avventura in un giambico ${ }^{22}$, ora si stempera in un sonoro alcaico ${ }^{23}$, ora scivola nel saffico ${ }^{24}$, ora batte il passo con il metro di mezzo piede ${ }^{25}$ ? Che cosa mai può essere più bello del cantico del Deuteronomio ${ }^{26}$ e di Isaia ${ }^{27}$ ? Che cosa più grave delle parole di Salomone $^{28}$ ? Che cosa più raffinato di Giobbe ${ }^{29}$ ? Tutte queste opere, come

${ }^{20}$ Quinto Orazio Flacco (Quintus Horatius Flaccus; nato a Venosa [in latino: Venusia] nel nordest della Basilicata, l'8 dicembre 65 a.C., e morto a Roma, il 27 novembre 8 a.C.), è stato uno dei maggiori poeti latini dell'età antica.

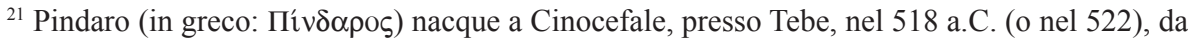
una nobile famiglia originaria della Beozia. Morì ad Argo, tra il 445 e il 438 a.C. Scrisse importanti carmi epici, viaggiò a lungo e scrisse per sovrani e famiglie importanti.

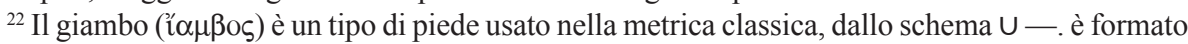

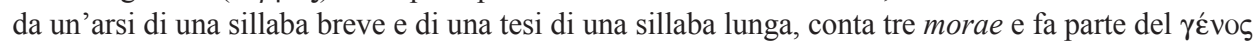

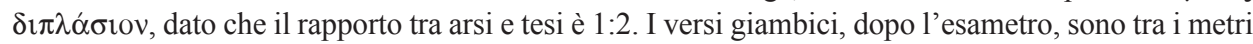
greci più antichi. Essi furono utilizzati nella poesia giambica e nella metrica corale, e continuarono ad essere adoperati sia nella poesia alessandrina che in quella latina. Nell'età classica, inoltre, il trimetro giambico divenne il metro consueto delle parti parlate della tragedia e della commedia, e il modello da cui gli antichi romani trassero il senario giambico.

${ }^{23}$ Strofa alcaica è una strofa composta da due endecasillabi alcaici, un enneasillabo alcaico e un decasillabo alcaico. Essa viene attribuita ad Alceo, il celebre poeta di Lesbo. La strofa alcaica fu largamente utilizzata anche nella letteratura latina, specialmente da Orazio nelle sue Odi (Carmina).

${ }^{24}$ Strofa saffica è una strofa composta da tre endecasillabi saffici e da un adonio. Essa viene attribuita alla celebre poetessa di Lesbo, Saffo. La "saffica" fu ampiamente utilizzata anche nella letteratura latina, specialmente da Catullo.

${ }^{25}$ Nella metrica classica il piede (in greco: $\pi 0 \hat{\varsigma} \varsigma$, in latino: pes; chiamato in tal modo perché il ritmo si batteva con il piede), era composto da un insieme di due o più sillabe brevi e lunghe che formavano la misura del verso. Nel piede ci sono due elementi: uno forte (detto "arsi" e segnato dall'ictus) e uno più debole (chiamato "tesi", dove la voce si abbassava). Un piede era composto almeno da due sillabe e tre morae.

${ }^{26} \mathrm{Si}$ tratta della benedizione (inno) di Mosè alle Dodici tribù di Israele (cf. Dt 33, 1-29).

${ }^{27} \mathrm{Il}$ profeta Isaia fu anche un grande poeta. Infatti, il suo libro è uno dei più poetici dell'Antico Testamento e possiede numerosi cantici (cf. ad esempio: Is 5, 1-2; 9, 1-6; 12, 1-6; 26, 1-12; 33, 2-10. $13-16$; 38, 10-20; $401-17$; 42, 10-16; 45, 15-25; 49, 7-13; 61, 6 - 62, 7; 63, 1-5; 66, 10-14).

${ }^{28} \mathrm{Al}$ re Salomone vengono attribuiti due Salmi (Sal 72 e Sal 127), la maggior parte del libro dei Proverbi (Pr 1 - 22, 16 e Pr 25-29), l'Ecclesiaste o Qoelet, il Cantico dei Cantici. Il suo regno è datato circa dal 970 al 930 a.C.

${ }^{29} \mathrm{Il}$ Libro di Giobbe è scritto in ebraico. La redazione del nucleo poetico centrale risale all'XI-X secolo a.C. La redazione definitiva, però, con le aggiunte in prosa (prologo ed epilogo) è stata fatta in Giudea verso il 575 a.C. 
scrivono Giuseppe $^{30}$ e Origene ${ }^{31}$, furono composte in esametri ${ }^{32}$ e pentame$\operatorname{tri}^{33}$, e in questa forma circolano presso la loro stessa gente. Quando li leggiamo in greco, essi hanno un certo significato; quando li leggiamo in latino risultano profondamente incoerenti. Ma se qualcuno ritiene che la grazia di una lingua non soffra nella traduzione, lasciagli tradurre in latino, parola per parola, Omero ${ }^{34}$. Vi dirò di più: se costui traducesse l'autore nella prosa della sua stessa lingua, l'ordine delle parole apparirebbe ridicolo, e il più eloquente dei poeti, un eloquente a stento" 35 .

Nel terzo capitolo dell'introduzione alla sua traduzione dell'opera eusebiana, perciò, san Girolamo si giustifica, mettendo in risalto, ancora una volta, tantissime difficoltà che accompagnano ogni traduzione:

"Quale è il senso di tutto questo? Non vorrei che pensaste che è strano se qui e là noi inciampassimo; se la lingua va a rilento; se pullula di consonanti o presenta lacunosi chiasmi di vocali, o fosse bloccata da condensazioni

${ }^{30}$ Flavio Giuseppe (in latino: Titus Flavius Iosephus), nato a Gerusalemme, nel 37 circa, e morto a Roma, nel 100 circa, fu scrittore, storico, politico e militare romano di origine ebraica; compose le sue opere in greco.

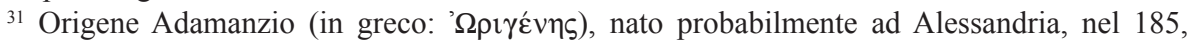
e morto a Tiro, nel 254), è uno tra i principali scrittori, filosofi, filologi biblici e teologi cristiani nei primi tre secoli.

${ }^{32}$ L'esametro dattilico o esametro eroico, spesso detto "esametro", è il più antico ed importante dei metri della poesia greca e latina (usato specialmente nella poesia epica o didattica). L'esametro, verso eroico per antonomasia, rimase strettamente legato alla poesia epica. Accanto alla poesia epica, l'esametro divenne, a partire da Esiodo, il metro della poesia didascalica. Grazie alle opere di Teocrito e di Virgilio, l'esametro divenne anche il metro della poesia bucolica. Esso fu invece raramente utilizzato nella poesia lirica.

${ }^{33}$ Il pentametro dattilico o "pentametro", è una forma metrica della poesia greca e latina. Esso è un metro composto, formato da due hemiepes, o tripodie dattiliche catalettiche. Il nome "pentametro" deriva dal fatto di essere la somma di due unità da due piedi e mezzo; dato però che è un metro dattilico, di ritmo discendente, il pentametro conta sei tesi o tempi forti. Il pentametro si incontra anche nella poesia drammatica, o talvolta è stato impiegato in versi stichici, ma il suo utilizzo principale si vede nel distico elegiaco, dove compare come secondo verso a seguito di un esametro. Il distico elegiaco è unito anzitutto a due generi letterari, strettamente legati tra di loro: l'elegia e l'epigramma.

${ }^{34}$ Omero (in greco: "O $\mathrm{n} \eta \rho \varsigma$ ) è il nome con con il quale viene identificato il poeta greco autore dell'Iliade e dell'Odissea - i due massimi poemi epici della letteratura greca antica.

${ }^{35}$ Hieronymus, Praefatio in Eusebii Caesariensis Chronicon 2, PL 27, 36-37: "Denique quid Psalterio canorius, quod in morem nostri Flacci, et Graeci Pindari nunc iambo currit, nunc alcaico personat, nunc Sapfico tumet, nunc senipede ingreditur. Quid Deuteronomii et Esaiae Cantico pulchrius, quid Solomone gravius, quid perfectius Iob. Quae omnia hexametris et pentametris versibus, ut Iosephus et Origenes scribunt, apud suos composita decurrunt. Haec cum Graeca legimus, aliud quiddam sonant, cum Latine, penitus non haerent. Quodsi cui non videtur linguae gratiam interpretatione mutari, Homerum ad verbum exprimat Latinum, - plus aliquid dicam - eundem in sua lingua prosae verbis interpretetur: videbit ordinem ridiculum et poetam eloquentissimum vix loquentem". 
della narrazione. I più dotti fra gli uomini hanno sudato sette camicie nell'impresa $[\ldots]^{\prime 36}$.

La traduzione viene resa maggiormente difficile a causa dei "barbarismi" e dei concetti che sono estranei alla mentalità dei Latini:

"[...] in aggiunta alla difficoltà che tutti sperimentiamo, e che abbiamo associato all'impresa della traduzione, non va dimenticato che un'ulteriore difficoltà ci assale, nella misura in cui la storia è molteplice, è piena di nomi barbari, circostanze di cui i Latini non conoscevano nulla, dato che sono aggrovigliati nodi, momenti critici che si mescolano con gli eventi e i numeri; così che è persino più difficile discernere la sequenza delle parole che giungere alla comprensione di quanto è riferito" ${ }^{\prime 37}$.

Il brano che segue, sempre dello stesso terzo capitolo, fornisce indicazioni tecniche riguardanti i colori ed altri mezzi con i quali il Dalmata distribuì graficamente il testo tradotto. Purtroppo, per noi tutto ciò rimane quasi del tutto incomprensibile, dato che non possediamo l'archetipo dell'opera geronimiana:

"Per questo, credo che si debba avvertire che, così come ogni brano è scritto, anche si debba conservare la diversità dei colori, affinché nessuno ritenga che, per un gradimento irrazionale, si sia soltanto cercata un'idea delle cose occulte e che per fuggire dalla noia si sia imboccato il labirinto dell'errore. Ciò è stato pensato per il motivo che le espansioni dei regni, che per la loro eccessiva vicinanza quasi si sovrapponevano, fossero separate da una punteggiatura in minio e nello stesso posto del colore segnalato nella prima pergamena, si conservasse anche la scrittura successiva" ${ }^{38}$.

Conformemente al suo temperamento e anche alle regole retoriche, san Girolamo conclude la Praefatio apostrofando tutti coloro che potrebbero criticare e detrarre la sua traduzione e, specialmente, la sua continuazione della Cronaca:

${ }^{36}$ Ibidem 3, PL 27, 37: "Quorsum ista? Videlicet ut non vobis mirum videatur, si alicubi offendimus, si tarda oratio aut consonantibus asperatur aut vocalibus hiulca fit aut rerum ipsarum brevitate constringitur, cum eruditissimi homines in eodem opere sudaverint $[\ldots]$ ”.

${ }^{37}$ Ibidem: "[...] et ad communem difficultatem, quam in omni interpretatione causati sumus, hoc nobis proprium accedat, quod historia multiplex est habens barbara nomina, res incognitas Latinis, numeros inextricabiles, virgulas rebus pariter ac numeris intertextas, ut paene difficilius sit legendi ordinem discere quam ad lectionis notitiam pervenire".

${ }^{38}$ Ibidem, PL 27, 38: "Unde praemonendum puto, ut, prout quaeque scripta sunt, etiam colorum diversitate serventur, ne quis inrationabili aestimet voluptate oculis tantum rem esse quaesitam et, dum scribendi taedium fugit, labyrinthum erroris intexat. Id enim elucubratum est, ut regnorum tramites, qui per vicinitatem nimiam paene mixti erant, distinctione minii separarentur et eundem coloris locum, quem prior membrana signaverat, etiam posterior scriptura servaret”. 
"Sono del tutto consapevole che vi saranno molti che, con la loro consueta passione per la detrazione universale (la cui unica fuga consiste nel non scrivere nulla), affonderanno i denti in questo volume, eccepiranno cavilli nelle date, cambieranno l'ordine, impugneranno l'accuratezza degli eventi, separeranno le sillabe e, come spesso accade, imputeranno la negligenza dei copisti agli autori. Mi sento in diritto di respingerli dicendo che non debbono necessariamente leggere l'opera a meno che non lo vogliano; ma preferirei allontanarli con calma, in modo che possano prestare il debito credito all'autore greco e riconoscere che qualsiasi inserimento di cui siamo responsabili è stato preso da altri uomini degni della più grande stima" ${ }^{\text {"39. }}$.

Prioprio alla fine del terzo capitolo della Praefatio, san Girolamo precisa il metodo ed alcuni particolari tecnici concernenti la traduzione:

"La verità è che io ho parzialmente rifiutato l'ufficio di traduttore e parzialmente quello di scrittore. Ho reso, con la più grande fedeltà, la porzione greca e, allo stesso tempo, aggiunto alcune cose che mi sembravano concesse, specialmente riguardo la storia romana, su cui Eusebio, l'autore di questo libro, per quanto mi è parso, ha solo sorvolato; non per ignoranza, in quanto era dotto, ma perché, scrivendo in greco, li ritenne di poca importanza - per i suoi [compaesani]. Pertanto, ancora una volta, da $\mathrm{Nino}^{40}$ ad $\mathrm{Abramo}^{41}$, fino alla presa di Troia ${ }^{42}$, la traduzione è solo dal greco. Da Troia fino al ventesimo

${ }^{39}$ Ibidem, PL 27, 38-39: "Nec ignoro multos fore, qui solita libidine omnibus detrahendi huic volumini genuinum infigant, quod vitare non potest nisi qui omnino nil scribit: calumniabuntur in tempora, convertent ordinem, res arguent, syllabas eventilabunt et, quod accidere plerumque solet, negligentiam librariorum ad auctores referent. Quos cum possem meo iure repercutere, ut, si displicet, non legant, malo breviter placatos dimittere, ut et Graecorum fidem suo auctori assignent et quae nova inservimus de aliis probatissimis viris libata cognoscant".

${ }^{40} \mathrm{Fu}$ re assiro e marito della mitica Semiramide. Divenne un personaggio letterario a cominciare dal periodo ellenistico. Gli venne attribuita la fondazione della città di Ninive che da lui prese il nome. Alcune leggende lo presentano figlio del Dio Baal che conquistò in 17 anni tutta l'Asia occidentale con l'aiuto di Ariaeo, re dell'Arabia, fondando il primo impero assiro. Durante l'assedio di Bactria, Nino conobbe Semiramide, all'epoca moglie di un suo ufficiale, che lo fece innamorare e divenne sua moglie. Ella gli partorì la figlia Ninia. Dopo la morte di Nino, Semiramide fu accusata di esserne stata la causa, ma ella, per difendersi, fece erigere per lui un magnifico mausoleo-tempio, vicino a Babilonia. Secondo Castore, il regno di Nino durò 52 anni, dal 2189 a.C. (secondo lo storico greco Ctesias).

${ }^{41}$ Il primo patriarca della vera religione, Abramo visse tra il XX e il XIX secolo a.C. Di lui, e degli eventi con lui collegati, parla il Libro della Genesi (cf. Gn 11, 27 - 25, 10).

42 Verso la fine dell'età del Bronzo, intorno 1300-1200 a.C. I particolari del conflitto sono noti primariamente grazie all'Iliade e all'Odissea di Omero, composte intorno al IX secolo a.C.: l'Iliade narra fatti avvenuti durante l'ultimo anno di guerra, l'Odissea, invece, il viaggio di Ulisse per tornare in patria, dopo la presa della città. La storicità della guerra di Troia è ancora oggi oggetto di discussione. Alcuni studiosi ritengono che i racconti di Omero siano l'unione di diversi conflitti scoppiati tra greci e troiani nel periodo miceneo. La maggior parte dei greci antichi pensava che la guerra di Troia fosse un fatto realmente accaduto, altri ritenevano che Omero avesse ingigantito a scopi poetici un avvenimento non famoso come quello descritto. Nel 1870 gli studiosi concor- 
anno di Costantino ${ }^{43}$ c'è molto, aggiunto separatamente in un determinato periodo e in modo mescolato in un altro periodo, che io ho messo insieme con grande diligenza da Tranquillo ${ }^{44}$ e altri famosi storici" ${ }^{45}$.

San Girolamo precisa anche quale parte di quest'opera egli scrisse personalmente, continuando quella eusebiana:

"Inoltre, la parte dal sopracitato anno di Costantino al sesto consolato dell'imperatore Valente e al secondo di Valentiniano ${ }^{46}$ è interamente mia. Lieto di concludere qui, ho riservato il periodo restante, quello di Graziano ${ }^{47}$ e di Teodosio ${ }^{48}$, ad una più ampia disamina; non che io sia timoroso di scrivere dei viventi liberamente e in verità, perché il timore di Dio bandisce il timore degli uomini, ma perché, mentre il nostro paese è ancora esposto alla furia dei barbari, ogni cosa è confusa" ${ }^{\prime 4}$.

davano sul fatto che la guerra di Troia non fosse mai accaduta. Attualmente, molti studiosi sono d'accordo sul fatto che la guerra di Troia sia un fatto realmente accaduto, dubitano però sul fatto che gli scritti di Omero raccontino fedelmente la sua vicenda.

${ }^{43}$ Flavio Valerio Aurelio Costantino, conosciuto anche come Costantino il Grande, o Costantino I (Flavius Valerius Aurelius Constantinus), nato a Naissus (città romana, situata lungo il fiume Nišava, nell'odierna Serbia, dove oggi sorge la città di Niš), il 27 febbraio 274, e morto a Nicomedia, 22 maggio 337, fu imperatore romano dal 306 alla sua morte.

${ }^{44}$ Gaio Svetonio Tranquillo (Caius Suetonius Tranquillus), nato in un luogo imprecisato del Latium vetus, forse ad Ostia o addirittura a Roma, attorno al 70, e morto in una località a noi sconosciuta, attorno al 126, o al 140, è uno scrittore romano d'età imperiale, principale esponente del genere della biografia. Fu un erudito e un ricercatore encicolpedico, paragonabile a Marco Terenzio Varrone. Le due principali opere di Svetonio sono: il De viris inlustribus, e il De vita Caesarum.

${ }^{45}$ Hieronymus, Praefatio in Eusebii Caesariensis Chronicon 3, PL 27, 39-40: "Sciendum etenim est me et interpretis et scriptoris ex parte officio usum, quia et Graeca fidelissime expressi et nonnulla, quae mihi intermissa videbantur, adieci, in Romana maxime historia, quam Eusebius huius conditor libri non tam ignorasse ut eruditus, sed ut Graece scribens parum suis necessariam perstrinxisse mihi videtur. Itaque a Nino et ab Abraham usque ad Troiae captivitatem pura Graeca translatio est. A Troia usque ad vicesimum Constantini annum nunc addita, nunc admixta sunt plurima, quae de Tranquillo et ceteris inlustribus historicis curiosissime excerpsi”.

${ }^{46}$ Il sesto consolato dell'imperatore Valente (364-378) fu nell'anno 378. Il 378 fu anche l'anno del secondo consolato dell'imperatore Valentiniano II (375-392).

${ }^{47}$ Flavio Graziano (Flavius Gratianus), nato a Sirmio, il 18 aprile/23 maggio 359, morto a Lione, il 25 agosto 383, fu imperatore romano dal novembre 375 alla sua morte.

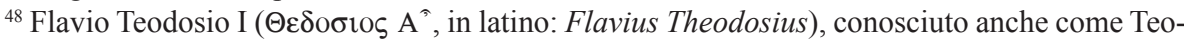
dosio I, nato a Cauca (l'odierna Coca in Spagna), l'11 gennaio 347, e morto a Milano, il 17 gennaio 395, è stato un imperatore romano dal 379 fino alla sua morte.

${ }^{49}$ Hieronymus, Praefatio in Eusebii Caesariensis Chronicon 3, PL 27, 40: "A Constantini autem supra dicto anno usque ad consulatum Augustorum Valentis sexies et Valentiniani iterum totum meum est. Quo fine contentus reliquum temporis Gratiani et Theodosii latioris historiae stilo reservavi, non quo de viventibus timuerim libere et vere scribere: timor enim Dei hominum timorem expellit; sed quoniam dibacchantibus adhuc in terra nostra Barbaris incerta sunt omnia". 
Con queste parole, finisce la prefazione gerominiana alla traduzione della Cronaca di Eusebio di Cesarea. Dopo la traduzione, san Girolamo continuerà la Cronaca, a partire dal 326:

"Fino a questo punto, Eusebio di Panfilo ${ }^{50}$, commilitone dei martiri ${ }^{51}$, scrive questa storia, alla quale noi abbiamo aggiunto quanto segue" $" 52$.

San Girolamo continuerà la Cronaca descrivendo diversi avvenimenti storici sino all'anno 378 (o forse 379) dopo Cristo, e terminando con queste parole:

"Valente, obbligato a lasciare Antiochia, con un pentimento tardivo richiama la nostra gente dall'esilio" 53 .

"Deplorevole guerra in Tracia. Le legioni romane, a causa della mancata difesa della cavalleria, vengono accerchiate dai Goti e massacrate. Lo stesso imperatore Valente, ferito da una freccia e, a causa di un grande dolore, cadento spesso dal cavallo, trova rifugio in un casolare dove viene raggiunto dai barbari che danno fuoco al nascondiglio. Egli non ottiene neanche sepoltura" ${ }^{54}$.

Basandoci sulla Prefazione genonimiana alla sua traduzione delle Cronache di Eusebio di Cesarea, oltre alle notizie riguardanti la stessa opera del vescovo di Cesarea in Palestina, possiamo anche conoscere meglio il metodo del Girolamo traduttore.

Il Dalmata evidenzia numerose difficoltà che si fanno sentire in queste ardue imprese che i traduttori come tali devono affrontare, essendo a voltre costretti a "storpiare" il testo originale per renderne il vero senso nella loro

${ }^{50}$ Eusebio di Cesarea (Palestina) (265ca. - 339). Suo maestro fu il martire Panfilo, il più colto degli allievi di Origene, per il quale Eusebio nutre stima così grande da assumerne il nome:

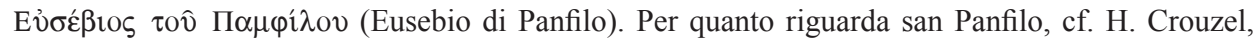
Panfilo di Cesarea, NDPAC III 3811-3812. Per Eusebio di Cesarea, cf. C. Curti, Eusebio di Cesarea (Palestina), NDPAC I 1845-1853.

${ }^{51}$ Viene qui usata la parola contubernalis che possiamo tradurre: "commilitone". Il contubernium era la più piccola unità militare dell'esercito romano.

${ }_{52}$ Hieronymus, Continuatio Chronicorum Eusebii Caesariensis A.D. 326, in: Scriptorum veterum nova collectio, ed. A. Maio, VIII, Romae 1883, 400: "Huc usque historiam scribit Eusebius Pamphyli martyris contubernalis. Cui nos ista subiecimus".

${ }^{53}$ Ibidem A.D. 378, ed. Maio, p. 405: "Valens de Antiochia exire conpulsus sera paenitentia nostros de exiliis revocat".

${ }^{54}$ Ibidem A.D. 379, ed. Maio, p. 405: "Lacrimabile bellum in Thracia, in quo deserente equitum praesidio Romanae legiones a Gothis cinctae usque ad internecionem caesae sunt. Ipse imperator Valens, cum sagitta saucius fugeret et ob dolorem nimium saepe equo laberetur, ad cuiusdam villulae casam deportatus est, quo persequentibus barbaris et incensa domo sepultura quoque caruit". 
propria lingua. Lo stesso testo da tradurre è, di regola, difficile da capire, quindi anche da essere trasformato in un'altra lingua.

Le difficoltà diventano maggiori se si tratta di testi di stampo poetico. Infatti, se si traducesse un poeta in prosa, l'ordine delle parole apparirebbe ridicolo e persino incomprensibile. San Girolamo si giustifica, quindi, mettendo in risalto tantissime difficoltà che accompagnano ogni traduzione.

La traduzione spesso viene resa maggiormente difficile a causa dei "barbarismi" e dei concetti che sono estranei alla mentalità dei Latini. Il Dalmata, perciò, apostrofa tutti coloro che potrebbero criticare e detrarre la sua traduzione e, in un certo senso, anche le traduzioni di coloro che affrontano una simile ed ardua impresa.

\section{PREFACJA ŚW. HIERONIMA DO KONTYNUACJI KRONIKI EUZEBIUSZA Z CEZAREI}

\section{(Streszczenie)}

Zanim Euzebiusz z Cezarei napisał swoje najsławniejsze dzieło Historia

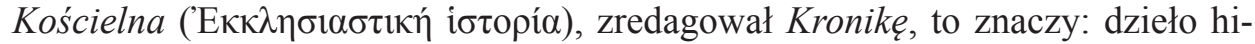
storyczne, które streszcza dzieje świata. Grecki oryginał tego dzieła zaginął, ale mamy całe jego thumaczenie w języku armeńskim i częściowe jego tłumaczenie po łacinie, którego dokonał właśnie św. Hieronim.

Po przedstawieniu w pierwszej części syntezy historii wschodniej i greckiej, w drugiej części dzieła Euzebiusz prezentuje tablice, które przedstawiają historię ludzkości od Abrahama (2016 prz. Chr.) do roku 303 po Chr., i właśnie ta druga część Kroniki Euzebiusza została przetłumaczona przez św. Hieronima. To tłumaczenie św. Hieronim uzupełnił dochodząc do roku 378 po Chr. Hieronim przeczytał Kronikę Euzebiusza i przetłumaczył ją mniej więcej w roku 380. Zrobił to podczas swojego pobytu w Konstantynopolu.

Pomimo wielu błędów i nieścisłości, które św. Hieronim przejął z tekstu greckiego dzieła Euzebiusza, ale także pomimo błędów popełnionych przez samego św. Hieronima, to historyczne dzieło jest użyteczne także i dzisiaj. W przeszłości natomiast zostało ono wykorzystane przez starożytnych historiografów, którzy je dalej kontynuowali. Dla przykładu byli to: Prosper z Akwitanii, Kasjodor, czy też Wiktor z Tunnuny.

Key words: Eusebius of Caesarea, Saint Jerome, translations, ancient history.

Parole chiave: Eusebio di Cesarea, san Girolamo, traduzioni, storia antica.

Słowa kluczowe: Euzebiusz z Cezarei, św. Hieronim, tłumaczenia, historia starożytna. 